\title{
Degree Distribution in Quantum Walks on Complex Networks
}

\author{
Mauro Faccin, ${ }^{1, *}$ Tomi Johnson, ${ }^{1,2}$ Jacob Biamonte, ${ }^{1}$ Sabre Kais, ${ }^{3,4}$ and Piotr Migdal ${ }^{5,1}$ \\ ${ }^{1}$ Institute for Scientific Interchange, Via Alassio 11/c, 10126 Torino, Italy \\ ${ }^{2}$ Clarendon Laboratory, University of Oxford, Parks Road, Oxford OX1 3PU, United Kingdom \\ ${ }^{3}$ Department of Chemistry, Physics, and Birck Nanotechnology Center, Purdue University, West Lafayette, Indiana 47907, USA \\ ${ }^{4}$ Qatar Environment and Energy Research Institute (QEERI), Doha, Qatar \\ ${ }^{5}$ ICFO-Institut de Ciències Fotòniques, 08860 Castelldefels (Barcelona), Spain \\ (Received 29 May 2013; revised manuscript received 1 August 2013; published 24 October 2013)
}

\begin{abstract}
In this theoretical study, we analyze quantum walks on complex networks, which model network-based processes ranging from quantum computing to biology and even sociology. Specifically, we analytically relate the average long-time probability distribution for the location of a unitary quantum walker to that of a corresponding classical walker. The distribution of the classical walker is proportional to the distribution of degrees, which measures the connectivity of the network nodes and underlies many methods for analyzing classical networks, including website ranking. The quantum distribution becomes exactly equal to the classical distribution when the walk has zero energy, and at higher energies, the difference, the socalled quantumness, is bounded by the energy of the initial state. We give an example for which the quantumness equals a Rényi entropy of the normalized weighted degrees, guiding us to regimes for which the classical degree-dependent result is recovered and others for which quantum effects dominate.
\end{abstract}

DOI: 10.1103/PhysRevX.3.041007

\section{INTRODUCTION}

A quantum walk on a network is a fundamental natural process [1-5] since the quantum dynamics of any discrete system can be reexpressed and interpreted as a singleparticle quantum walk, which is capable of performing universal quantum computation [6]. Quantum walks are also of increasing relevance outside physics. As well as being a powerful tool for studying transport in quantum systems [7-10], e.g., the transport of energy through biological complexes or man-made solar cells, quantum walks have been proposed as a means of analyzing classical sociological networks [11-14]. Fully understanding these phenomena, and others, for networks with nontrivial topologies, requires the merging of the methods of complex networks and quantum mechanics [15].

While analytical results have been obtained for some specific topologies, such as starlike [16-18], regular, or semiregular [19] networks, progress in analyzing quantum walks on complex networks has largely been based on numerics, leaving open the possibility that many conclusions are not representative of all regimes. In this article, we instead discover analytical properties of continuoustime unitary quantum walks of arbitrary topology that follow from the topology of the underlying complex network.

\footnotetext{
*mauro.faccin@isi.it
}

Published by the American Physical Society under the terms of the Creative Commons Attribution 3.0 License. Further distribution of this work must maintain attribution to the author(s) and the published article's title, journal citation, and DOI.
Subject Areas: Complex Systems, Quantum Physics

A widely applicable analysis of unitary quantum walks has remained illusive because of a strong dependence on the initial state that is exacerbated by the lack of convergence to a steady state (which is not necessarily the case for open quantum walks $[20,21]$ ). To overcome these difficulties and obtain a result that is relevant beyond specific initial states and walk durations, we consider a quantity that characterizes each walk over long times and relate this to another quantity that characterizes each initial state. Specifically, we characterize a walk by the long-time average probability distribution of finding the walker at each node [16,22], which captures all knowledge of the location of the walker in the absence of knowledge about when the walk began. The initial state is naturally characterized by the energy, which gives a total ordering of the initial states. We show that for low energies the long-time average probability distribution is equal to the normalized distribution of degrees in the network. Specifically, the energy bounds the trace distance between the two distributions. This provides a wide class of quantum walks on complex networks with an analytically tractable low-energy regime.

Our result is achieved by mapping the properties of the ground state of a quantum walk to the steady state of a corresponding classical walk-in particular, a classical walk whose steady state represents the connectivity of nodes as determined by their degree. Such walks are used by search engines, e.g., Google, to rank websites $[23,24]$. Our result extends the importance of the concept of degree from classical systems [25-30], ranging from the sociological to the ecological [31-33], to quantum systems [7-10,34-36].

As a case study, we analytically and numerically study the walk for a range of model complex network 
structures, including the Barabàsi-Albert (BA), ErdôsRényi (ER), Watt-Strogatz (WS), and random geometric (RG) networks. We repeat this analysis for several realworld networks, specifically, a karate club (KC) social network [37], the email (EM) network of Universitat Rovira i Virgili (URV) [38], the C. elegans metabolic (CE) network [39], and a coauthorship (CA) network of scientists [40]. To compare these networks, we start from an evenly distributed initial state. We find an additional connection to the degrees for this case, namely, that the quantumness of the walk is itself controlled by the heterogeneity of the degrees, which we quantify in terms of a Rényi entropy.

In Sec. II, we formulate and study the problem analytically, first for a stochastic walk and then for a quantum walk. Following this, in Sec. III, we confirm our analytical results for the quantumness of a quantum walk numerically and explore the way in which the quantum long-time average deviates from the corresponding classical distribution. We conclude with a discussion in Sec. IV.

\section{WALKS FRAMEWORK}

We consider a walker moving on a connected network of $N$ nodes, with each weighted undirected edge between nodes $i$ and $j$ described by the element $A_{i j}$ of the offdiagonal adjacency matrix $A$. The matrix is symmetric $\left(A_{i j}=A_{j i}\right)$ and has real, non-negative entries. We use Dirac notation and represent $A=\sum_{i j} A_{i j}|i\rangle\langle j|$ in terms of $N$ orthonormal vectors $|i\rangle$.

The network gives rise to both a quantum walk and a corresponding classical walk. The classical stochastic walk $S(t)=\mathrm{e}^{-H_{C} t}$ is generated by the infinitesimal stochastic (see, e.g., Refs. [41-43]) operator $H_{C}=L D^{-1}$, where $L=D-A$ is the Laplacian and $D=\sum_{i} d_{i}|i\rangle\langle i|$ is defined by its diagonal elements, the degrees, $d_{i}=$ $\sum_{j} A_{i j}$. For this classical walk, the total rate of leaving each node is identical. The corresponding unitary quantum walk $U(t)=\mathrm{e}^{-\mathrm{i} H_{Q} t}$ is generated by the Hermitian operator $H_{Q}=D^{-1 / 2} L D^{-1 / 2}$. For this quantum walk, the energies $\left\langle i\left|H_{Q}\right| i\right\rangle$ at each node are identical. The generators $H_{C}$ and $H_{Q}$ are similar matrices, related by $H_{Q}=$ $D^{-1 / 2} H_{C} D^{1 / 2}$. This mathematical framework, represented in Fig. 1, underpins our analysis.

As we will describe in Sec. II A, the long-time behavior of the classical walk generated by $H_{C}$ has been well explained in terms of its underlying network properties, specifically, the degrees $d_{i}$. Our goal in Sec. II B is to determine the role this concept plays in the quantum walk generated by $H_{Q}$.

\section{A. Classical walks}

In the classical walk, the probability $P_{i}(t)$ of being at node $i$ at time $t$ evolves as $|P(t)\rangle=S(t)|P(0)\rangle$, where $|P(t)\rangle=\sum_{i} P_{i}(t)|i\rangle$. The stationary states of the walk are described by eigenvectors $\left|\pi_{i}^{k}\right\rangle$ of $H_{C}$ with eigenvalues $\lambda_{i}$

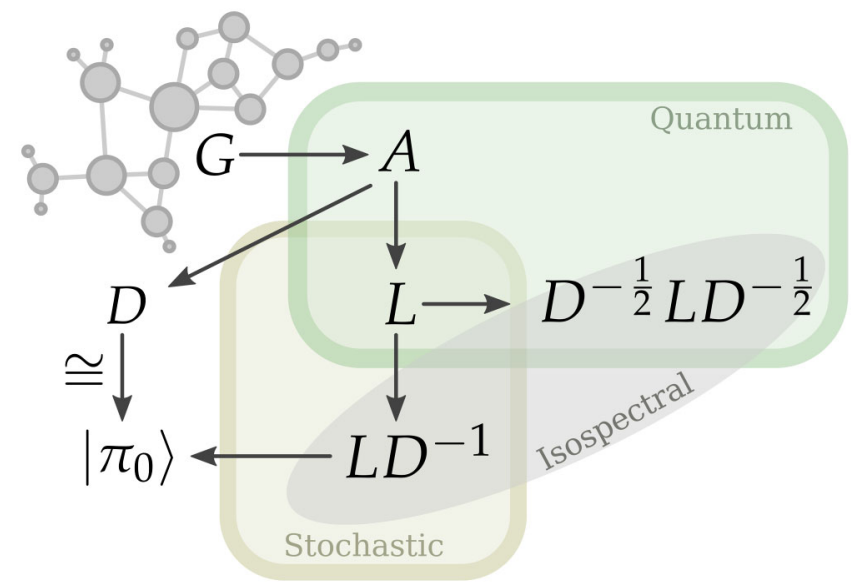

FIG. 1. Relating stochastic and quantum walks. An undirected weighted network (graph) $G$ is represented by a symmetric, offdiagonal and non-negative adjacency matrix $A$. There is a mapping from $A$ (by summing columns) to the diagonal matrix $D$, with entries given by the weighted degree of the corresponding node. The node degrees are proportional to the steady-state probability distribution of the continuous-time stochastic walk (with uniform escape rate from each node) generated by $H_{C}=$ $L D^{-1}$, where $L=D-A$ is the Laplacian. The steady-state probabilities, represented by the vector $\left|\pi_{0}\right\rangle$, are proportional to the node degrees. We generate a corresponding continuoustime unitary quantum walk by the Hermitian operator $H_{Q}=$ $D^{-1 / 2} L D^{-1 / 2}$, which is similar to $H_{C}$. The probability of being in a node in the stochastic stationary state $\left|\pi_{0}\right\rangle$ and the probability arising from the quantum ground state are equal and proportional to the node degree.

equal to zero. We assume throughout this work that the walk is connected, i.e., that it is possible to transition from any node to any other node through some series of allowed transitions. In this case, there is a unique eigenvector $\left|\pi_{0}\right\rangle=\left|P_{C}\right\rangle$ with $\lambda_{0}=0$, and $\lambda_{i}>0$ for all $i \neq 0[41,44-46]$. This (normalized) eigenvector $\left|P_{C}\right\rangle=$ $\sum_{i}\left(P_{C}\right)_{i}|i\rangle$ describes the steady-state distribution

$$
\left(P_{C}\right)_{i}=\frac{d_{i}}{\sum_{j} d_{j}}
$$

In other words, the process is ergodic, and after long times, the probability of finding the walker at any node $i$ is given purely by the importance of the degree $d_{i}$ of that node in the network underlying the process.

\section{B. Quantum walks}

When considering quantum walks on networks, it is natural to ask what the long-time behavior of a quantum walker is $[11,15,22,47]$. The unitary evolution will not drive the system towards a steady state. Therefore, to obtain a static picture, we consider the long-time average probability $\left(P_{Q}\right)_{i}$ of being on node $i$, which reads

$$
\left(P_{Q}\right)_{i}=\lim _{T \rightarrow \infty} \frac{1}{T} \int_{0}^{T} \mathrm{~d} t\left\langle i\left|U(t) \rho(0) U^{\dagger}(t)\right| i\right\rangle .
$$


For ease of comparison with $\left|P_{C}\right\rangle$, we will also write the distribution in Eq. (3) as a ket $\left|P_{Q}\right\rangle=\sum_{i}\left(P_{Q}\right)_{i}|i\rangle$. Unlike the classical case, Eq. (2) depends on the initial state $\rho(0)$.

Interference between subspaces of different energy vanishes in the long-time average, so we obtain an expression for the probability $\left(P_{Q}\right)_{i}$ in terms of the energy-eigenspace projectors $\Pi_{j}$ of the Hamiltonian $H_{Q}$,

$$
\left(P_{Q}\right)_{i}=\sum_{j}\left\langle i\left|\Pi_{j} \rho(0) \Pi_{j}\right| i\right\rangle .
$$

Here, $\Pi_{j}=\sum_{k}\left|\phi_{j}^{k}\right\rangle\left\langle\phi_{j}^{k}\right|$ projects onto the subspace spanned by the eigenvalues $\left|\phi_{j}^{k}\right\rangle$ of $H_{Q}$, corresponding to the same eigenvalue $\lambda_{j}$. In other words, the long-time average distribution is a mixture of the distributions obtained by projecting the initial state onto each eigenspace.

Because of the similarity transformation $H_{Q}=$ $D^{-1 / 2} H_{C} D^{1 / 2}$, the classical $H_{C}$ and quantum $H_{Q}$ generators share the same eigenvalues $\lambda_{i} \geq 0$ and have eigenvectors related by $\left|\phi_{i}^{k}\right\rangle=D^{-1 / 2}\left|\pi_{i}^{k}\right\rangle$ up to their normalizations. In particular, the unique eigenvectors corresponding to $\lambda_{0}=0$ are $\left|\pi_{0}\right\rangle=D|\mathbf{1}\rangle$ and $\left|\phi_{0}\right\rangle=D^{1 / 2}|\mathbf{1}\rangle$ up to their normalizations, with $|\mathbf{1}\rangle=\sum_{i}|i\rangle$. Therefore, the probability vector describing the outcomes of a measurement of the quantum ground-state eigenvector $\left|\phi_{0}\right\rangle$ in the node basis is the classical steady-state distribution $\left|\pi_{0}\right\rangle=\left|P_{C}\right\rangle$.

The state vector $\left|P_{C}\right\rangle$ appears in Eq. (3) for the quantum long-time average distribution $\left|P_{Q}\right\rangle$ with weight $\left\langle\phi_{0}|\rho(0)| \phi_{0}\right\rangle$. Accordingly, we split the sum in Eq. (3) into two parts: The first part we call the "classical term" $\left|P_{C}\right\rangle$, and the second we call the "quantum correction" $\left|\tilde{P}_{Q}\right\rangle$, given as

$$
\left|P_{Q}\right\rangle=(1-\varepsilon)\left|P_{C}\right\rangle+\varepsilon\left|\tilde{P}_{Q}\right\rangle .
$$

The normalized quantum correction $\left|\tilde{P}_{Q}\right\rangle=\sum_{i}\left(\tilde{P}_{Q}\right)_{i}|i\rangle$ is given by

$$
\left(\tilde{P}_{Q}\right)_{i}=\frac{1}{\varepsilon} \sum_{j \neq 0}\left\langle i\left|\Pi_{j} \rho(0) \Pi_{j}\right| i\right\rangle,
$$

and the weight

$$
\varepsilon=1-\left\langle\phi_{0}|\rho(0)| \phi_{0}\right\rangle
$$

that we call quantumness is a function of both the degrees, through $\left|\phi_{0}\right\rangle$, and the initial state.

We can think of the parameter $\varepsilon$, which controls the classical-quantum mixture, as the quantumness of $\left|P_{Q}\right\rangle$ for the following three reasons. First, the proportion of the elements in $\left(P_{Q}\right)_{i}$ that corresponds to the quantum correction is $\varepsilon$. Second, the trace distance between the normalized distribution $\left(P_{C}\right)_{i}$ and the unnormalized distribution $(1-\varepsilon)\left(P_{C}\right)_{i}$ forming the classical part of the quantum result is also $\varepsilon$. Last, using a triangle inequality, the trace distance between the normalized distributions $\left(P_{C}\right)_{i}$ and $\left(P_{Q}\right)_{i}$ is upper bounded by $2 \varepsilon$.
This expression for the quantumness in Eq. (6) enables us to make some physical statements about a general initial state. By realizing that $\left|\phi_{0}\right\rangle$ is the ground state of zero energy $\lambda_{0}=0$ and the gap $\Delta=\min _{i \neq 0} \lambda_{i}$ in the energy spectrum is nonzero for a connected network [41,44-46], the above implies a bound $E / \Delta \geq \varepsilon$ for the quantumness $\varepsilon$ of the walk in terms of the energy $E=\operatorname{tr}\left\{H_{Q} \rho\right\}$ of the initial state. The bound is obtained through the following steps:

$$
\begin{aligned}
E & =\operatorname{tr}\left\{H_{Q} \rho\right\}=\sum_{j \neq 0} \lambda_{j} \operatorname{tr}\left\{\Pi_{j} \rho(0)\right\} \geq \Delta \sum_{j \neq 0} \operatorname{tr}\left\{\Pi_{j} \rho(0)\right\} \\
& =\Delta\left(1-\operatorname{tr}\left\{\Pi_{0} \rho(0)\right\}\right)=\Delta \epsilon .
\end{aligned}
$$

The equation above demonstrates that the classical stationary probability distribution will be recovered for low energies. A utility of this result is that it connects the longtime average distribution to a simple physical property of the walk, the energy, which provides a total ordering of all possible initial states.

\section{Degree distribution and quantumness}

Quantumness is a function of both the degrees of the network nodes and the initial state. To compare the quantumness of different complex networks, we fix the initial state $\rho(0)$. For our example, we choose the even superposition state $\rho(0)=|\Psi(0)\rangle\langle\Psi(0)|$ with $|\Psi(0)\rangle=|\mathbf{1}\rangle / \sqrt{N}$. This state has several appealing properties; for example, it is invariant under node permutations and independent of the arrangement of the network.

In this case, the quantumness is given by the expression

$$
\varepsilon=1-\frac{\langle\sqrt{d}\rangle^{2}}{\langle d\rangle}
$$

where $\langle d\rangle=\sum_{i} d_{i} / N$ is the average degree and $\langle\sqrt{d}\rangle=$ $\sum_{i} \sqrt{d_{i}} / N$ is the average root degree of the nodes. As such, the quantumness depends only on the degree distribution of the network and increases with network heterogeneity.

This statement is quantified by writing the quantumness

$$
\varepsilon=1-\frac{1}{N} \exp \left[H_{1 / 2}\left(\left\{\frac{d_{i}}{\sum_{j} d_{j}}\right\}\right)\right]
$$

in terms of the Rényi entropy

$$
H_{q}\left(\left\{p_{i}\right\}\right)=\frac{1}{1-q} \ln \left(\sum_{i} p_{i}^{q}\right),
$$

where $d_{i} / \sum_{j} d_{j}=\left(P_{C}\right)_{i}$ are the normalized degrees.

To obtain an expression in terms of the (perhaps) more familiar Shannon entropy $H_{1}$ [obtained by taking the $q \rightarrow 1$ limit of Eq. (10)], we recall that the Rényi entropy is nonincreasing with $q$ [48], which leads to the upper bound

$$
\varepsilon \leq 1-\frac{1}{N} \exp \left[H_{1}\left(\left\{\frac{d_{i}}{\sum_{j} d_{j}}\right\}\right)\right]
$$


The quantumness approaches this upper bound in the limit that $M$ nodes have uniform degree $d_{i}=M\langle d\rangle / N$ and all others have $d_{i}=0$. This limit is never achieved unless $M=N$ and $\varepsilon=0$, e.g., in a regular network. Physically, $\varepsilon=0$ for a regular network because the symmetry of the Hamiltonian $H_{Q}$ implies that its eigenvectors are evenly distributed. The only eigenvector of this type that is positive is the initial state $|\Psi(0)\rangle$, which because of the PerronFrobenius theorem, must also be the ground state $|\Psi(0)\rangle=$ $\left|\phi_{0}\right\rangle$. Therefore, $E=0$ and so, from Eq. (7), $\varepsilon=0$.

In another limit, the quantumness takes its maximum value $\varepsilon=(N-2) / N \approx 1$ when the degrees of two nodes are equal and much larger than those of the others (note that the symmetry of $A$ prevents the degree of a single node from dominating). In the case that $A_{i j} \in\{0,1\}$, i.e., the network underlying the walks is not weighted, the quantumness of a connected network is more restricted. It is maximized by a walk based on a star network-where a single node is connected to all others. For a walk of this type, $\varepsilon=1 / 2-\sqrt{N-1} / N \approx 1 / 2$.

Next, in Sec. III, we confirm the above analytical findings numerically and, at the same time, numerically study the form of the quantum correction $\left|\tilde{P}_{Q}\right\rangle$ given by Eq. (5) for a range of complex network topologies.

\section{NUMERICAL RESULTS}

\section{A. Artificial network topologies}

We consider walkers on model networks, each with a fundamentally different complex network topology. To start, we consider nonweighted binary networks $A_{i j} \in$ $\{0,1\}$ with $N=500$ nodes and average degree $\langle d\rangle \approx 6$. If

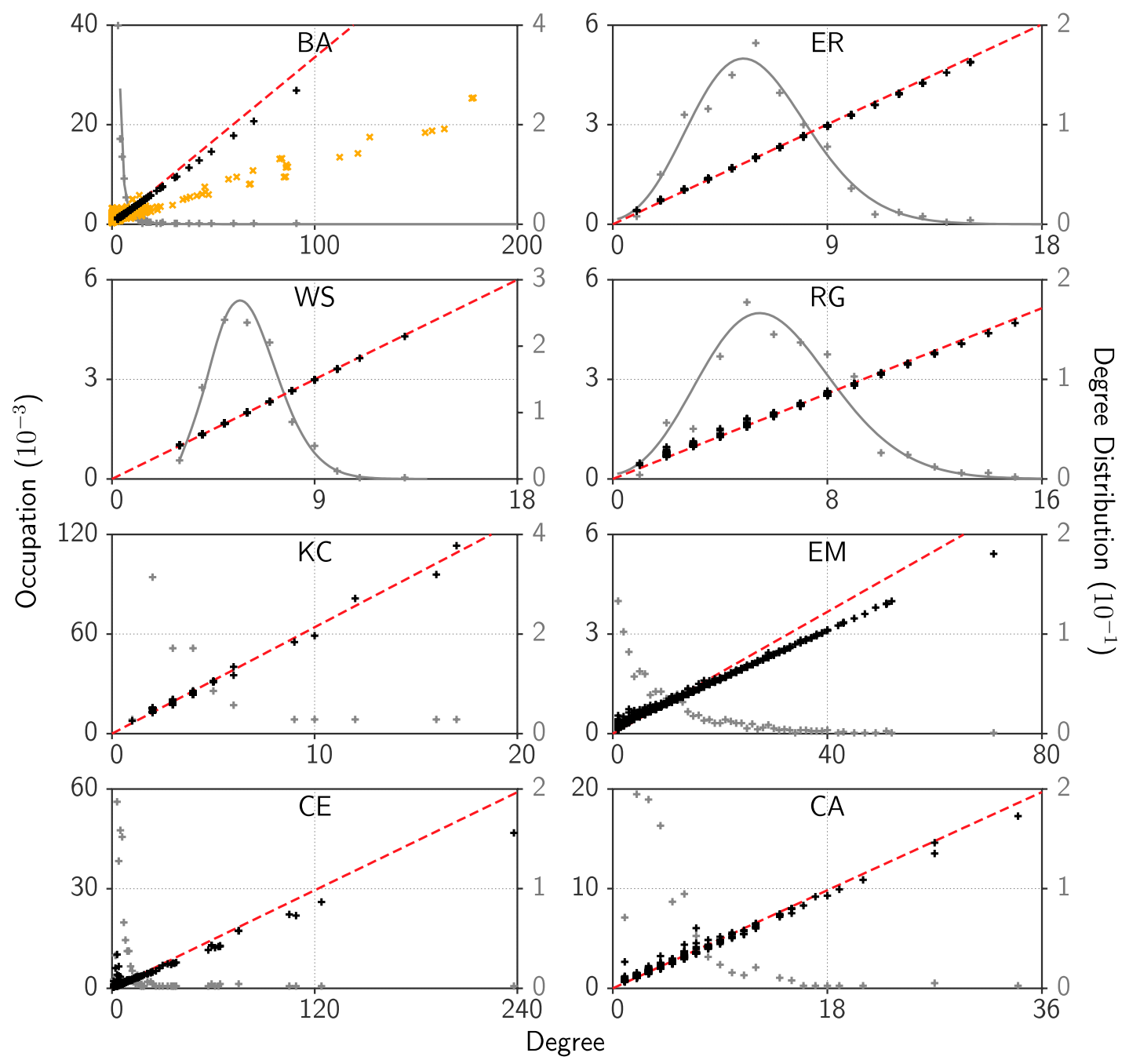

FIG. 2. Long-time average probability and degree for nodes in a complex network. Eight networks are considered: BA, ER, WS, RG, $\mathrm{KC}, \mathrm{EM}, \mathrm{CE}$, and CA. We plot the classical $\left(P_{C}\right)_{i}$ (red dashed line) and quantum $\left(P_{Q}\right)_{i}$ (black +$)$ probabilities against the degree $d_{i}$ for every node $i$. We overlay this with a plot of the average degree distribution $P(d)$ against $d$ for each network type (grey solid line), when known, along with the distribution for the specific realization used (grey +$)$. Alongside the BA network, we also plot $\left(P_{Q}\right)_{i}$ for the optimized BA (BA-opt) network, in which the internode weights of the BA network are randomly varied in a Monte Carlo algorithm to reach $\varepsilon=0.6$ (orange $\mathrm{x}$ ). We do not include a plot of the degree distribution for this network. 
a disconnected network is obtained, only the giant component is considered. Specifically, we consider the BA scale-free network [25], the ER [49] and the WS [50] smallworld networks, and the RG (on a square) [51], a network without the scale-free or small-world characteristics.

The long-time average probability of being on each node $i$ is plotted against its degree $d_{i}$ for a quantum $\left(P_{Q}\right)$ and a stochastic $\left(P_{C}\right)$ walk in Fig. 2 . The two cases are nearly identical for these binary networks and the evenly distributed initial state, illustrating that the quantumness $\varepsilon$ is small. We have in fact calculated the quantumness directly for each network type, yielding $\varepsilon=0.130,0.043,0.016$, and 0.040 for the BA, ER, WS, and RG networks, respectively. Within these, the BA network shows the highest quantum correction. This result is expected since the BA network has a more heterogeneous degree distribution. The WS network, which is well known to have quite uniform degrees [52], is accordingly the network with the lowest quantum correction.

For many of the network types, the typical quantumness can be obtained from the expected (thermodynamic limit) degree distribution. In the BA network, the degree distribution approximately obeys the continuous probability density $P(d)=\langle d\rangle^{2} / 2 d^{3}$ [25]. Integrating this to find the moments results in $\varepsilon=1 / 9$, which is independent of the average degree $\langle d\rangle$ and is compatible with our numerics. The degree distributions of the ER and RG networks both approximately follow the Poissonian distribution $P(d) \approx$ $\langle d\rangle^{d} \mathrm{e}^{-\langle d\rangle} / d$ ! for large networks, which explains the similarity of their quantumness $\varepsilon$ values. For $\langle d\rangle=6$, we recover $\varepsilon \approx 0.046$, which is compatible with the values for the particular networks we generated. From the general form, calculating the quantumness numerically and performing a best fit, we find that $\varepsilon \approx \kappa_{1}\langle d\rangle^{-\kappa_{2}}$, with fitting parameters $\kappa_{1}=0.429$ and $\kappa_{2}=1.210$.

The size of the quantum effects can be enhanced by introducing heterogeneous weights $A_{i j}$ within a network. We have done this for a BA network by using several iterations of the following procedure. A pair of connected nodes is randomly selected, and then the associated weight is doubled or halved at random. As anticipated, the effect is to increase the discrepancy between the classical and quantum dependence of the long-time average probability on degree, illustrated in Fig. 2. As the number of iterations is increased, the quantumness follows the bound given in Eq. (11), as shown in Fig. 3. In fact, most networks are found to be close to saturating this bound, especially for low quantumness.

Further, the energy $E=\left\langle\Psi_{0}\left|H_{Q}\right| \Psi_{0}\right\rangle$ of the given initial state has a simple expression $E=1-(1 / N) \times$ $\sum_{i j} A_{i j} / \sqrt{d_{i} d_{j}}$, which allows us to determine the extent to which the bound $E / \Delta \geq \varepsilon$ is saturated by comparing the values of $E / \Delta$ and $\varepsilon$. We find that for some networks, e.g., the BA, ER, and WS networks, the bound is quite restrictive and reasonably saturated. However, for the other networks, we find that quantumness takes a low

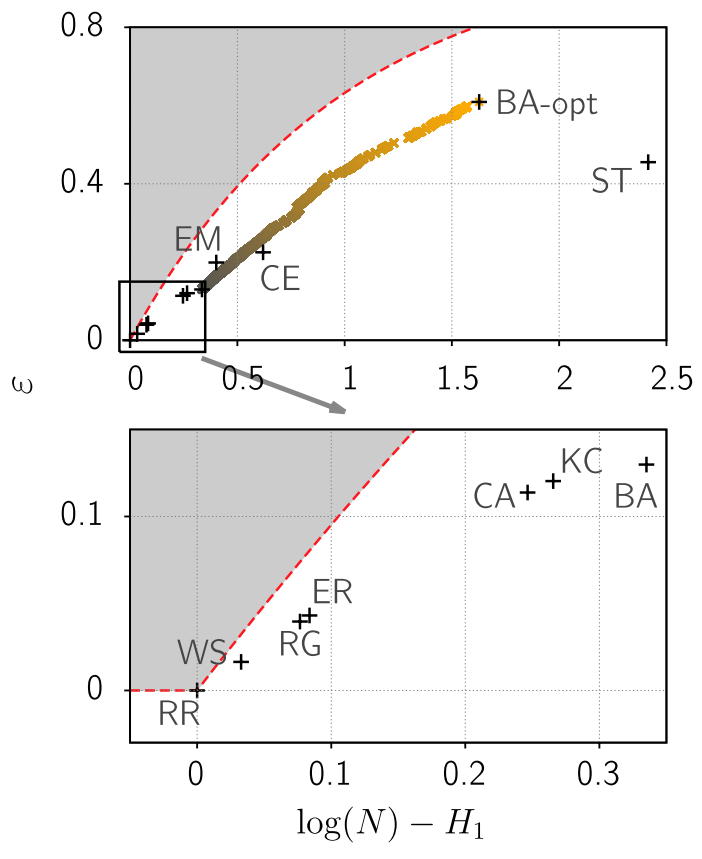

FIG. 3. Quantumness and degree entropy. The value of $\varepsilon$ against $H_{1}$ [Eq. (10)] for the nine different networks considered in Fig. 2, as well as the random regular (RR) network (a network with the same degree for each node-in this case, we consider a regular graph with each node of degree six) and star (ST) networks (black + ). We also plot $\varepsilon$ and $H_{1}$ for the network obtained in several iteration steps, each randomly varying an internode weight of the BA network, for an increasing number of iteration steps (bottom to top, grey to orange $\mathrm{x}$ ). The quantumness $\varepsilon$ increases and the entropy $H_{1}$ decreases with step number. The red dashed line represents the upper bound of Eq. (11).

value without being ensured by the bound only (see Table I).

Finally, our numerical calculations reveal the behavior of the quantum part $\tilde{P}_{Q}$ of the long-time average node occupation. We find that the quantum part enhances the long-time average probability of being at nodes with small degree relative to the classical part. More precisely,

TABLE I. Quantumness, energy, and gap. We show the quantumness $\varepsilon$ and its upper bound $E / \Delta$ (the ratio of the energy and the gap), for each of the nine networks considered in Fig. 2.

\begin{tabular}{lcr}
\hline \hline Type & $\varepsilon$ & \multicolumn{1}{c}{$E / \Delta$} \\
\hline BA & 0.1299 & 0.5583 \\
ER & 0.0431 & 0.1734 \\
RG & 0.0396 & 11.2875 \\
WS & 0.0164 & 0.0846 \\
BA-opt & 0.6092 & 844.9181 \\
KC & 0.1204 & 1.3471 \\
CE & 0.2247 & 4.7622 \\
EM & 0.1987 & 1.5449 \\
CA & 0.1138 & 39.8535 \\
\hline \hline
\end{tabular}


$\left(\tilde{P}_{Q}\right)_{i} /\left(P_{C}\right)_{i}$ exhibits roughly $\left(d_{i}\right)^{-\kappa_{3}}$ scaling, with $\kappa_{3} \approx 1$, as shown in Fig. 4. Interestingly, there is a correlation between the amount of enhancement, given by $\kappa_{3}$, and the type of complex network. The network types with smaller diameters (order of increasing diameter: BA, then ER and WS, then RG) have the smallest $\kappa_{3}$, and the quantum parts enhance the low-degree nodes least. Moreover, the enhancement $\kappa_{3}$ seems to be quite independent of the internode weights. Thus, our numerics show a qualitatively common quantum effect for a range of complex network types. Quantitative details vary between the network types but appear robust within each type.

\section{B. Real-world network topologies}

The models of networks examined in the previous subsection have very specific topologies and, therefore, degree distributions, and they do not capture the topological properties of all real-world networks (for details, see Chap. 9 of
Ref. [30]). Therefore, we now study the behavior of the quantumness and gap for topologies present in a variety of real-world networks, as described in Sec. I: a karate club (KC) social network [37], the e-mail (EM) network of URV University [38], the C. elegans metabolic (CE) network [39], and a coauthorship (CA) network of scientists [40].

The values of the quantumness and comparison against the entropic upper bound are shown in Fig. 3. Despite the variety of topologies, we again find that the quantumness is consistently small. Therefore, the classical and quantum distributions are very close, as shown in Fig. 2. Additionally, the quantum correction exhibits the same generic behavior as observed for the artificial networks; Fig. 4 shows an enhancement of the probability of being in nodes of small degree. Interestingly, the quantumness of real-world networks is appreciably smaller than enforced by the bound of Eq. (7), with $E / \varepsilon \Delta$ taking large values, as shown in Table I.
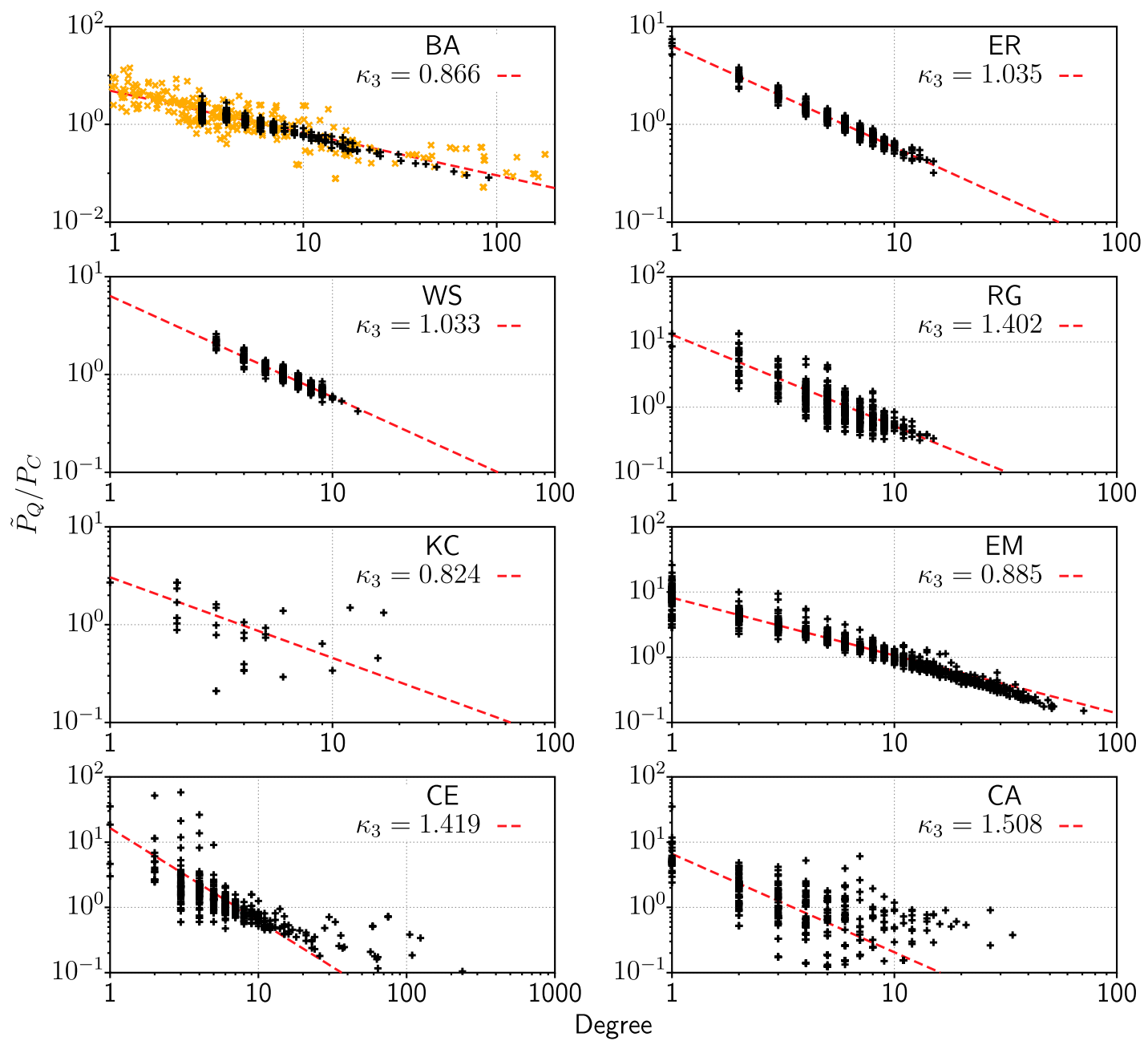

FIG. 4. Quantum effects. The ratio of the quantum $\left(\tilde{P}_{Q}\right)_{i}$ and classical $\left(P_{C}\right)_{i}$ probabilities plotted against degree $d_{i}($ black +$)$ for every $i$, for the networks considered in Fig. 2. We also plot the best-fitting curve (red dashed line) to these data of the form $\left(\tilde{P}_{Q}\right)_{i} /\left(P_{C}\right)_{i} \propto$ $\left(d_{i}\right)^{\kappa_{3}}$, whose exponent $\kappa_{3}$ is given in the plot. 


\section{DISCUSSION}

We have found an analytical expression for the average long-time probability distribution for the location of a lowenergy quantum walker on a complex network of arbitrary topology. Specifically, we have shown that this is equal to the distribution arising in the steady state of a corresponding classical walk, equal to the normalized degrees. As well as providing an analytical solution for low-energy walks, our result will allow the benchmarking of other methods for studying quantum walks on complex networks, a field in which numerical analysis is typically the only viable option.

The stationary state of the classical walk generated by the asymmetrically normalized Laplacian $H_{C}$ is closely connected to the ranking of nodes within a network, as used by Google. Therefore, our results indicate that the long-time average probability distribution of a quantum walk under $H_{Q}$, with the energy of the initial state as a free parameter, could provide a means of interpolating between classical and quantum [11,12] ranking of real-world networks. This idea also connects nicely with the work of Ref. [14] in which the authors numerically simulate driving a quantum system to its ground state, with the quantum system chosen such that its ground state represents the Google ranking vector.

For the evenly distributed initial state, the quantumness (loosely speaking, the difference between the classical and quantum distributions) only depends on the degrees. Together with our result for the low-energy distribution, this result shows that the degree distribution can be as important and illuminating in quantum walks as in their classical counterparts. Our numerical examples also show that for remarkably diverse network types, quantum effects are qualitatively similar; they act to reduce the degree dependence of the average probability of a walker being found on a node.

Our presentation focused on a walker state $\rho$ that is a time average over a unitary evolution. However, to conclude, note that our analytical solution to the expected node occupation $\langle i|\rho| i\rangle$ holds whenever a significant portion of $\rho$ is in the ground-state subspace of $H_{Q}$, i.e., when $\epsilon=$ $1-\Pi_{0} \rho \Pi_{0}$ is small. To see this, one can always use $\sum_{j} \Pi_{j}=1$ to expand $\langle i|\rho| i\rangle=(1-\varepsilon)\left(P_{C}\right)_{i}+\varepsilon\left(\tilde{P}_{Q}\right)_{i}$. Similarly, the bound $E=\operatorname{tr}\left\{H_{Q} \rho\right\} \geq \varepsilon \Delta$ will always hold [Eq. (7)]. In particular, these results are independent of whether $\rho$ is obtained by a unitary or a nonunitary walk. For example, the steady state $\rho$ of a walker equilibrating with a low-temperature bath has a small $\varepsilon$; thus, $\langle i|\rho| i\rangle$ is proportional to the degree.

\section{ACKNOWLEDGMENTS}

We thank Michele Allegra, Ville Bergholm, Stephen Kirkland, Giovanni Petri, and Simone Severini for fruitful discussions. J.D.B. would like to thank the Qatar
Environment and Energy Research Institute (QEERI), where part of this research was completed. P. M. would like to acknowledge the Spanish MINCIN/MINECO project TOQATA (FIS2008-00784), EU Integrated Projects AQUTE and SIQS, and HISTERA project DIQUIP.

[1] Martí Cuquet and John Calsamiglia, Entanglement Percolation in Quantum Complex Networks, Phys. Rev. Lett. 103, 240503 (2009).

[2] S. Perseguers, M. Lewenstein, A. Acín, and J.I. Cirac, Quantum Random Networks, Nat. Phys. 6, 539 (2010).

[3] S. Perseguers, D. Cavalcanti, G. J. Lapeyre, M. Lewenstein, and A. Acín, Multipartite Entanglement Percolation, Phys. Rev. A 81, 032327 (2010).

[4] Richard P. Feynman, Robert B. Leighton, and Matthew Sands, The Feynman Lectures on Physics (AddisonWesley, Reading, MA, 2005), 2nd ed.

[5] Richard P. Feynman and A. R. Hibbs, Quantum Mechanics and Path Integrals, International Series in Pure and Applied Physics (McGraw-Hill, New York, 1965).

[6] Andrew M. Childs, Universal Computation by Quantum Walk, Phys. Rev. Lett. 102, 180501 (2009).

[7] E. Farhi and S. Gutmann, Quantum Computation and Decision Trees, Phys. Rev. A 58, 915 (1998).

[8] F. Caruso, A. W. Chin, A. Datta, S. F. Huelga, and M. B. Plenio, Highly Efficient Energy Excitation Transfer in Light-Harvesting Complexes: The Fundamental Role of Noise-Assisted Transport, J. Chem. Phys. 131, 105106 (2009).

[9] M. Mohseni, P. Rebentrost, S. Lloyd, and A. AspuruGuzik, Environment-Assisted Quantum Walks in Photosynthetic Energy Transfer, J. Chem. Phys. 129, 174106 (2008).

[10] Yuan-Chung Cheng and Graham R. Fleming, Dynamics of Light Harvesting in Photosynthesis, Annu. Rev. Phys. Chem. 60, 241 (2009).

[11] E. Sánchez-Burillo, J. Duch, J. Gómez-Gardeñes, and D. Zueco, Quantum Navigation and Ranking in Complex Networks, Nat. Sci. Rep. Ochanomizu Univ. 2, 605 (2012).

[12] G.D. Paparo and M. A. Martin-Delgado, Google in a Quantum Network, Sci. Rep. 2, 444 (2012).

[13] Silvano Garnerone, Paolo Zanardi, and Daniel A. Lidar, Adiabatic Quantum Algorithm for Search Engine Ranking, Phys. Rev. Lett. 108, 230506 (2012).

[14] Silvano Garnerone, Thermodynamic Formalism for Dissipative Quantum Walks, Phys. Rev. A 86, 032342 (2012).

[15] O. Mülken and A. Blumen, Continuous-Time Quantum Walks: Models for Coherent Transport on Complex Networks, Phys. Rep. 502, 37 (2011).

[16] Oliver Muelken, Inefficient Quantum Walks on Networks: The Role of the Density of States, arXiv:0710.3453.

[17] Oliver Mülken, Veronika Bierbaum, and Alexander Blumen, Coherent Exciton Transport in Dendrimers and Continuous-Time Quantum Walks, J. Chem. Phys. 124, 124905 (2006). 
[18] Chengzhen Cai and Zheng Yu Chen, Rouse Dynamics of a Dendrimer Model in the $\vartheta$ Condition, Macromolecules 30, 5104 (1997).

[19] S. Salimi, Continuous-Time Quantum Walks on SemiRegular Spidernet Graphs via Quantum Probability Theory, Quantum Inf. Process. 9, 75 (2010).

[20] Herbert Spohn, An Algebraic Condition for the Approach to Equilibrium of an Open N-Level System, Lett. Math. Phys. 2, 33 (1977).

[21] James D. Whitfield, César A. Rodríguez-Rosario, and Alán Aspuru-Guzik, Quantum Stochastic Walks: A Generalization of Classical Random Walks and Quantum Walks, Phys. Rev. A 81, 022323 (2010).

[22] Oliver Mülken, Antonio Volta, and Alexander Blumen, Asymmetries in Symmetric Quantum Walks on TwoDimensional Networks, Phys. Rev. A 72, 042334 (2005).

[23] Michalis Faloutsos, Petros Faloutsos, and Christos Faloutsos, On Power-Law Relationships of the Internet Topology, SIGCOMM Comput. Commun. Rev. 29, 251 (1999).

[24] R. Albert, H. Jeong, and A. L. Barabási, Internet: Diameter of the World-Wide Web, Nature (London) 401, 130 (1999).

[25] A.-L. Barabási and R. Albert, Emergence of Scaling in Random Networks, Science 286, 509 (1999).

[26] Réka Albert and Albert-László Barabási, Statistical Mechanics of Complex Networks, Rev. Mod. Phys. 74, 47 (2002).

[27] M. E. J. Newman, The Structure and Function of Complex Networks, SIAM Rev. 45, 167 (2003).

[28] Mark Newman, Networks: An Introduction (Oxford University Press, New York, NY, 2010).

[29] D. J. de Solla Price, Networks of Scientific Papers, Science 149, 510 (1965).

[30] Ernesto Estrada, The Structure of Complex Networks: Theory and Applications (Oxford University Press, New York, NY, 2011).

[31] Stanley Wasserman and Katherine Faust, Social Network Analysis. Methods and Applications (Cambridge University Press, Cambridge, England, 1994).

[32] Duncan J. Watts, Peter Sheridan Dodds, and M. E. J. Newman, Identity and Search in Social Networks, Science 296, 1302 (2002).

[33] M.E.J. Newman, Spread of Epidemic Disease on Networks, Phys. Rev. E 66, 016128 (2002).

[34] Zoltan Zimboras, Mauro Faccin, Zoltan Kadar, James Whitfield, Ben Lanyon, and Jacob Biamonte, Quantum Transport Enhancement by Time-Reversal Symmetry Breaking, Sci. Rep. 3, 2361 (2013).

[35] J. Kempe, Quantum Random Walks: An Introductory Overview, Contemp. Phys. 44, 307 (2003).
[36] Salvador Elias Venegas-Andraca, Quantum Walks for Computer Scientists, Synthesis Lectures on Quantum Computing 1, 1 (2008).

[37] Wayne W. Zachary, An Information Flow Model for Conflict and Fission in Small Groups, J. Anthropol. Res. 33, 452 (1977).

[38] Roger Guimera, Leon Danon, A Diaz-Guilera, Francesc Giralt, and Alex Arenas, Self-Similar Community Structure in a Network of Human Interactions, Phys. Rev. E 68, 065103 (2003).

[39] Jordi Duch and Alex Arenas, Community Detection in Complex Networks Using Extremal Optimization, Phys. Rev. E 72, 027104 (2005).

[40] Mark E.J. Newman, Finding Community Structure in Networks Using the Eigenvectors of Matrices, Phys. Rev. E 74, 036104 (2006).

[41] John C. Baez and Jacob Biamonte, A Course on Quantum Techniques for Stochastic Mechanics, arXiv:1209.3632.

[42] T. H. Johnson, S. R. Clark, and D. Jaksch, Dynamical Simulations of Classical Stochastic Systems Using Matrix Product States, Phys. Rev. E 82, 036702 (2010).

[43] J.C. Baez and B. Fong, A Noether Theorem for Markov Processes, J. Math. Phys. (N.Y.) 54, 013301 (2013).

[44] Joel Keizer, On the Solutions and the Steady States of a Master Equation, J. Stat. Phys. 6, 67 (1972).

[45] Peter Lancaster and Miron Tismenetsky, Theory of Matrices (Academic Press, New York, 1985), Vol. 2.

[46] James R. Norris, Markov Chains, 2008 (Cambridge University Press, Cambridge, England, 1998).

[47] Dorit Aharonov, Andris Ambainis, Julia Kempe, and Umesh Vazirani, Quantum Walks on Graphs, in Proceedings of the 33rd Annual ACM Symposium on Theory of Computing (Organization ACM, New York, NY, 2001), pp. 50-59.

[48] C. Beck and F. Schögl, Thermodynamics of Chaotic Systems: An Introduction (Cambridge University Press, Cambridge, England, 1993).

[49] P. Erdôs and A. Rényi, On the Evolution of Random Graphs, in Publication of the Mathematical Institute of the Hungarian Academy of Sciences (Hungarian Academy of Science, Budapest, Hungary, 1960), pp. 17-61.

[50] D. J. Watts and S.H. Strogatz, Collective Dynamics of "Small-World" Networks, Nature (London) 393, 440 (1998).

[51] Mathew Penrose, Random Geometric Graphs (Oxford University Press on Demand, Oxford, England, 2003), Vol. 5.

[52] Alain Barrat and M. Weigt, On the Properties of Small-World Network Models, Eur. Phys. J. B 13, 547 (2000). 